\title{
Reshaping Cell Line Development and CMC Strategy for Fast Responses to Pandemic Outbreak
}

\author{
Zheng Zhang ${ }^{1}$, Ji Chen ${ }^{1}$, Junghao Wang ${ }^{1}$, Qiao Gao ${ }^{1}$, Shurong Xu ${ }^{1}$, Li Zhang ${ }^{1}$, Jill Cai ${ }^{1}$, \\ and Weichang Zhou ${ }^{1}$ \\ ${ }^{1} \mathrm{WuXi}$ Biologics
}

November 27, 2020

\begin{abstract}
The global pandemic outbreak, SARS-COV-2, which causes COVID-19, has coerced numerous pharmaceutical companies to sprint for the vaccine and therapeutic biologics development. Most of the therapeutic biologics are common human IgG antibodies, which were identified by next-generation sequencing with the B cells from the convalescent patients in less than one-month post-infection. While the global public health emergency calls for medications urgently, it saves lives to expedite the clinical trials of biologics as much as possible, hence the biologics development strategies are unprecedentedly challenged. Since the advent of therapeutic biologics, transfection, and selection strategy has been continuously improving for developing more robust cell lines with greater productivity and efficiency. Next-generation sequencing (NGS) has also been implemented into cell bank testing for acceleration. These recent advances enable us to rethink and reshape the chemistry, manufacturing and controls (CMC) strategy against the pandemic outbreaks, to start supplying cGMP materials for the life-saving clinical trials as soon as possible. We elucidated an accelerated $\mathrm{CMC}$ workflow for biologics against pandemics, including using cGMP-compliant pool materials for Phase I clinical trials, selecting the final clone with similar product quality as Phase I materials for late-stage development and commercial production and matching product quality among different manufacturing stages.
\end{abstract}

Reshaping Cell Line Development and CMC Strategy for Fast Responses to Pandemic Outbreak

Authors

Dr. Zheng Zhang, Dr. Ji Chen, Dr. Junghao Wang, Qiao Gao, Shurong Xu, Li Zhang, Jill Cai, Dr. Weichang Zhou*

299 Fute Zhong Road, Waigaoqiao Free Trade Zone, WuXi Biologics, Shanghai, 200131, China

Corresponding author : Dr. Weichang Zhou, CTO of WuXi Biologics,

Email: weichang_zhou@wuxibiologics.com

\section{Abstract}

The global pandemic outbreak, SARS-COV-2, which causes COVID-19, has coerced numerous pharmaceutical companies to sprint for the vaccine and therapeutic biologics development. Most of the therapeutic biologics are common human IgG antibodies, which were identified by next-generation sequencing with the $\mathrm{B}$ cells from the convalescent patients in less than one-month post-infection. While the global public health emergency calls for medications urgently, it saves lives to expedite the clinical trials of biologics as much as possible, hence the biologics development strategies are unprecedentedly challenged. Since the advent of therapeutic biologics, transfection, and selection strategy has been continuously improving for developing more robust cell lines with greater productivity and efficiency. Next-generation sequencing (NGS) has also been implemented into cell bank testing for acceleration. These recent advances enable us to rethink 
and reshape the chemistry, manufacturing and controls (CMC) strategy against the pandemic outbreaks, to start supplying cGMP materials for the life-saving clinical trials as soon as possible. We elucidated an accelerated CMC workflow for biologics against pandemics, including using cGMP-compliant pool materials for Phase I clinical trials, selecting the final clone with similar product quality as Phase I materials for latestage development and commercial production and matching product quality among different manufacturing stages.

\section{Keywords:}

COVID-19, CMC for biologics, mammalian cell line development, next-generation sequencing, site-specific integration

\section{Introduction}

In the past several decades, outbreaks of epidemics, such as SARS, Ebola, MERS, Zika virus, and the still rampant COVID-19, have become surprisingly more frequent, posing an egregious threat to public health and safety. Despite the challenges in developing biologics in a shorter time than usual, the high efficacy and accuracy in neutralizing and containing pathogens have made these biologics more and more favored by both academic research and the pharmaceutical industry (Baum et al., 2020; Brouwer et al., 2020; Cao et al., 2020; Gao et al., 2020; Hansen et al., 2020; Ju et al., 2020; Lan et al., 2020; Nyon et al., 2018; Pinto et al. 2020; Rogers et al., 2020; Shi et al., 2020; Wang et al., 2020; Zhang et al., 2020). This change of preference would be quite unrealistic without vast experiences of developing countless biologics, as witnessed by the global pharmaceutical industry (Li et al., 2010; Kunert et al., 2016). However, even though there have been many different types of CMC strategies in accommodating all kinds of biologics development scenarios, the currently fastest CMC timeline from DNA to IND of 10- or 12-months are yet insufficient against the challenge of a global public health emergency. How to conduct the CMC activities with greater time-efficiency without compromising product quality while meeting regulatory requirements has put both the biologics development projects and the business interests under test (Sempowski et al., 2020).

Traditional CMC activities contain cell line development (CLD) and process development, including upstream, downstream, formulation, and analytical method development before toxicology and GMP production. To find the most appropriate clone from hundreds or thousands of clone candidates, usually, at least 4 months are required for exhaustive screening efforts during cell line development. Meanwhile, process development dedicated to the specific molecules, including cell culture process, purification process, formulation, and assay development, requires approximately 3 months before at least one round of process qualification with the final clone. To save these 3 months from painstaking developments and qualifications of process and assay, pandemic neutralizing antibody projects usually favor the molecules with less complexity, or the molecules with established platform architecture, since it is beneficial to take advantage of the platform process, which helps to avoid the complications of process or assay incompatibility and the uncertain time cost for the traditional tedious but indispensable developments and qualifications (Kelley, 2020; Bolisetty et al., 2020). Another recent advance in time-efficient strategy is by shifting the materials produced for pre-clinical toxicological study from by the selected top clone to an appropriate stable cell pool, which has been elaborated and exercised by several leading pharmaceutical companies (Bolisetty et al., 2020; Fan et al., 2017; Hu et al., 2017; Munro et al., 2017; Rajendra et al., 2017; Scarcelli et al., 2017; Wright et al., 2017). Typically, performing one round of limited-dilution cloning or FACS cloning with the subsequent clone selection requires at least 7 weeks beyond the establishment of a stable cell pool. Bringing the preclinical toxicology ahead of the traditional schedule replaces the speed-limiting activities to how to wisely unfold the CMC paths. With successful precedents of such practice, it is feasible to leverage the acceleration strategies with respect to the life-saving pre-clinical toxicological and Phase I first-in-human study against the global public health emergency. Because of the returning of the critical path to CMC, together with limited time for the process, assay, and formulation optimization, the beginning of a biologics CMC, cell line development, has a crucial significance for the subsequent manufacturing planning and decisions, which is also the perspective from which we prefer to rethink and reshape the CMC strategies. 
Here we described a series of key steps to accelerating the overall CMC timeline per the CLD scope. First of all, transfections were performed under cGMP conditions and recovered stable cell pools were used directly for toxicology and cGMP production. Second, high-throughput clone screening was implemented to obtain top clones, then thorough clone culturing evaluation and preliminary cell line stability study with these selected top clones were carried out in parallel. Next, the clone with the closest product quality from the culturing evaluation to the preceding cGMP pool material was selected as the final clone for master cell bank (MCB) creation and the manufacturing henceforward. Meanwhile, the platform process should be tested whenever possible for qualification and optimization with no time to squander.

\section{Materials and Methods}

\section{Cell Line Development}

CHO-K1 host cells that were originally purchased from ATCC and suspension-adapted by WuXi Biologics were maintained as a suspension culture in CD CHO medium (ThermoFisher Scientific) supplemented with $4 \mathrm{mM}$ L-glutamine, and incubated at 36.5 in a humidified $6 \% \mathrm{CO} 2$ in air (v/v) orbital shaking incubator at $150 \mathrm{rpm}$ with an orbital diameter of $50 \mathrm{~mm}$. The recombinant biologics-expressing cell lines were generated by transfecting vectors encoding recombinant genes by electroporation using a Bio-Rad Gene Pulser ${ }^{\text {TM }}$ (BioRad) in the cGMP facilities of WuXi Biologics. The transfectants were resuspended with CD CHO medium and returned to the shaking incubator. Cells were then sub-cultured and expanded accordingly every 3 days. A few weeks of sub-culturing are needed before compatible with fed-batch culturing for toxicology and GMP production.

Single-cell cloning was done by depositing single cells into microplates by FACSAria II ${ }^{\mathrm{TM}}$ after transfected cells recovered. Time-lapse images of each well were taken daily to monitor the growth of clones from the day of FACS sorting till three days after. Clonal colonies were expanded to 24 deep well plates or spin tubes, in which the cells were sub-cultured every three days, and seeded in AMBR 250 bioreactors for clone fed-batch culture screening.

\section{Fed-batch Culture}

Cells were seeded in basal media and fed with supplemental media based on the consumption of nutrients. Both the basal media and the supplemental media are commercially available. The viability and viable cell density were measured daily by Vi-Cell ${ }^{\mathrm{TM}}$ cell viability analyzers. The antibody titer was determined by Protein A HPLC with the harvested 14-day culture.

\section{Next-Generation Sequencing}

Viral DNA Screening: RNAs were extracted and reverse transcribed to generate cDNA, which was used for sequencing. WuXi Bio's proprietary program was used for data analysis.

\section{Product Quality Analysis}

The harvested cultures of fed-batch samples were purified by either one-step protein A chromatography capture (shake flask samples) or WuXi Biologics downstream platform (manufacturing samples). Purified samples were subjected to WuXi Biologics platform analytical methods, such as Size Exclusion Chromatography (SEC), imaged Capillary Isoelectric Focusing (iCIEF), and N-Glycan LC.

\section{Cell Line Stability}

After cells were expanded into spin tubes or shake flasks, selection pressure was removed for approximately 30 PDLs (population doubling levels) before fed-batch inoculation for the stability study.

In addition to the expression level, the single-cell $\mathrm{qPCR}$ method was also adopted on the candidates for top clones to probe the genetic stability as previously described (He et al., 2012).

The final cell line stability was performed with the MCB. 


\section{Using SSI Pools to Quickly Initiate Pandemic Molecule Manufacturing Campaigns for Phase I Clinical Trial}

Site-specific integration (SSI) technology has brought forward much greater efficiency in inserting genes of interest (GOIs) into the host cell genome than the classical random integration transfection methods. While SSI has greatly improved the productivity of the stable cell lines, the much higher efficiency of recombinant gene integration into the genome of the host cells than the traditional random insertion has even greater pragmatic value for the industry. While the traditional method of plating and screening of stable cell pools can take up to 3 months from transfection to the fed-batch culture for a material generation, the SSI stable pool takes as short as 3 weeks for the same scale of production. This enabled us to conceive a more aggressive but pragmatic CMC timeline for counter-pandemic projects like neutralizing antibody productions. Since the transfections are performed under cGMP conditions, the SSI transfectants can be directly expanded for the production of toxicology materials and even one or two following batches of GMP production (Gen1 GMP), depending on the calculated needs for the initial clinical trials. From the perspective of CLD, which is ultimately intended for the creation of a high-quality, monoclonal production cell line, prioritizing the closest product quality proximity to the Gen1 GMP materials will be necessary for the top clone selection criteria, to control the product quality consistency between the Gen1 GMP batch materials and the following clonal GMP batch materials (Gen2 GMP). The MCB of the final clone (Gen2 GMP path) will eventually be used to succeed the Gen1 GMP production for the subsequent supply of clinical materials. The high-level flowchart for such a strategy is illustrated in Fig. 1.

Our SSI pools have proven to be quite capable of productivity on several toxicology or GMP productions with COVID-19 molecules (Fig. 2A). One representative toxicology production, which was directly expanded after transfection, yielded harvested titer as high as $4 \mathrm{~g} / \mathrm{L}$ on day 14 with good growth and metabolic profiles, such as viability, viable cell density, glucose level, lactate level, and osmolality (Fig. 2B-F).

The comparability between toxicology and clinical materials is undoubtedly critical, one of the key challenges to overcome if the stable pools, instead of the monoclonal cell banks, should be used for manufacturing. The application of the SSI technology to such material generation strategy has an underlying prerequisite that the SSI pools should display stable product quality profiles, which we have observed with different seed train lengths for different batches of productions (data not shown). As illustrated in Figure 3, the toxicology batch and the following two GMP clinical batches of the same SSI pool (Molecule A) with different seed-train lengths shared similar productivity and product quality profiles to the smaller-scale bioreactor evaluations. In this case, the required amount of the appropriate materials was generated with satisfactory consistency, which also obviated the necessity for the purification and qualification protocol alterations.

\section{Selecting Clones from the SSI Pools with Similar Product Quality for Later Stage Development}

Although the materials from SSI pools have enabled the initiation of clinical material production immediately against the backdrop of pandemic outbreaks, clonal production cell lines are nevertheless required for laterstage process development and the subsequent greater-scale clinical and commercial manufacturing. With at least one batch of clinical materials generated by the Gen1 GMP campaign, the clonal production cell lines for the Gen2 GMP campaigns should particularly focus on the proximity of the product quality profiles along with the typical emphasis on productivity. For instance, there was one COVID-19 mAb molecule, whose transfectant cell pools were cloned in parallel with the Gen1 GMP production, to initiate the Gen2 development. From the clone screening results, the top clone candidates displayed a wide distribution of titer (Fig. 4A), as well as a broad kaleidoscope of glycan profiles (Fig. 4B) and charge variant profiles (Fig. $4 \mathrm{C})$. Such observation of widespread variety should be largely attributed to the diversity of the transfectant cells in the SSI pool, which provides excellent flexibility and convenience for clone selection to match Gen1 materials. The candidates with both the closest product quality attributes to the GMP materials and the higher titer were considered the final clone candidates for the following Gen2 activities, to which the process and assay used for Gen1 should be mostly applicable. With the platform process, similar product quality was obtained between Gen1 and Gen2 (Fig. 4D). 


\section{Adjusting Cell Line Stability Study to Unfold Final Clone Selection}

Traditional cell line stability study requires at least 60-generation cell passage and fed-batch evaluation between the pre-MCB (PCB) and the cells after stability passage (referred to as "PDL60"). The entire cell line stability takes almost 2 months, depending on the doubling time of the cell line. In the context of an aggressively accelerated CMC timeline, fitting in a full-length 12-week cell line stability could be particularly challenging. On the other hand, the instability of the final MCB clone, should it occur, could be devastating to the CMC plans. Therefore, however low risk of instability a cell line has, some degrees of the intelligence of the cell line stability should be acquired before deciding on the selection of the final MCB clone.

Empirically, loss of productivity usually occurs without selection pressure sustaining (data not shown). To evaluate the cell line stability as quickly as possible, selection pressure was removed as early as permissible by the actual needs of cells from other aspects, such as clone screening and bioreactor evaluation, and the cells were continuously passaged for approximately $30 \mathrm{PDLs}$ before fed-batch inoculation. Most of the clones displayed stable productivity (Fig. 5). A single-cell genetic stability test was also performed with the clone candidates to further probe the potential risks of cell line instability (He et al., 2012).

\section{Incorporating Next-Generation Sequencing to Accelerate Cell Bank Release with Mitigated Risks}

Biosafety of the cell banks is another important concern of the accelerated CMC strategy. Certain biosafety tests are required to control the risks before any cell banks are allowed to enter the GMP facilities. In a traditional CMC development, mycoplasma, typical viruses, retroviruses, and adventitious viruses are tested separately by more than ten different complicated assays. Even though not all the tests should be passed to release a cell bank into the GMP facility, mycoplasma, sterility, and 28-day in vitro virus testing are required to mitigate the risks of contamination, regardless. Although it takes only one or two weeks for relatively straightforward assays to conclude, some cellular assays, such as in vitro virus testing (IVV), could take several weeks to complete, which are too long to incorporate into an aggressively accelerated CMC strategy. On the other hand, given the current environmental control and extensive historical experience, the probability of biosafety failure is nearly negligible, enabling us to think about replacement tests for precautions.

To overcome this safety $v s$. time-efficiency dilemma, we performed an IVV test with the top 10 clone cell mixture and developed an NGS-based virus detection method (Khan et al., 2016 \& 2018; Brussel et al., 2019). For the proof of concept, we mixed serial proportions of mAb-expressing cells with the host cells, wherein we measured the RNA levels of the mAb. As shown in Table 1, the expression of mAb could still be detected when the sample contains only $1 \% \mathrm{mAb}$ expressing cells. Precedents are indicating that the virus will not grow at a composition of lower than 0.01 virus/cell (Richards et al., 2014; Wang, Feng and Duncan, 2014; Plavsic et al., 2016). Technically, we believe that the viral RNA can still be effectively detected even when the sample contains very low copies of the virus. For virus detection, the total RNA of the cell banks is analyzed by NGS and aligned to all available sequences in Reference Viral DataBase (RVDB). The virus contamination would be pronounced if the sequence coverage of any virus is higher than $50 \%$. This test can be considered as a precautionary test for early warnings, granting preliminary but sufficient support to permit the cell banks to enter the GMP facilities given the extremely low risks. However, all of the biosafety tests should still be performed in parallel to authenticate the NGS-based results, which is necessitated by the generation of subsequent working cell banks (WCB) and cGMP manufacturing. In short, cell banks can enter GMP facilities with NGS supported warrants with a turnaround time as short as within 1 week, which is also accredited to our refined algorisms.

\section{Case Studies}

The foregoing approach has been successfully incorporated into the CMC development of several molecules for pandemics at WuXi Biologics. Once the molecule sequence was received, a large-scale transient expression would be initiated either in parallel with or prior to the CLD (the beginning of Gen2 activities) and the seed train of toxicology batch and GMP production batch (Gen1 core activities). The materials from 
transient expressions were typically used for the evaluation and verification of the platform process and assay. Some alterations might be made to the process or assay accordingly to prepare for the upcoming Gen1 manufacturing campaigns. If any potential risky sequence of nucleotide or amino acid is identified in the complementarity-determining regions (CDRs), it will need to be altered, and the potency of the replacement sequences should be further evaluated by the live-virus assay with transient materials before unfolding the entire CMC landscape. The product quality attributes of two different COVID-19 molecules at different stages, on different scales (early transient batch, toxicology batch, GMP batch, and the Gen2 GMP batch) were shown in Fig. 6. By the platform process, the toxicology and GMP batches had quite consistent product quality attributes. Advanced CLD tools, such as FACS (Fluorescence Activated Cell Sorting) cloning, high throughput screening with 24-deep well plates, and single-cell qPCR for genetic stability were all used to obtain the most favorable clones for the MCBs. The top clone candidates displayed broad spectra of product quality profiles, especially for the charge variant and glycan profiles. The clone with the closest product quality attributes was selected for the MCB creation and future GMP productions. Judging from the product quality profiles in Fig. 6A-C, we managed to control the changes to a very narrow and acceptable range, especially between the GMP batches of Gen1 and Gen2, both the most strategically important materials. The consistent profiles of the product guaranteed smooth transition from the materials by SSI-pools to those by clones. In Fig. 6D-G, there are some other proven successful examples of CMC and manufacturing of different COVID-19 therapeutic molecules using such strategy, already en route to late-stage clinical studies.

\section{Discussion}

Nowadays, the pre-clinical toxicological study has been benefiting from the change of strategy to acquire materials directly from the SSI-transfectant cell pools. Against the backdrop of global pandemic outbreaks of COVID-19, this development further intrigues us with the possibility to generate materials for the Phase I clinical study from SSI-transfectant cell pools. To meet the safety requirements for the materials for the firstin-human clinical study, the entire Gen1 CMC campaign, including the transfection, seed train, cell banking, and manufacturing campaign should undoubtedly comply with the cGMP regulations. This strategy will rely heavily on the nature and origin of the neutralizing antibody; presumably, an antibody isolated from a convalescent patient with strong RBD (Receptor-Binding Domain) neutralizing activity should be more appropriate for such idea of Gen1 for clinical materials, which saves time from the complications introduced by other artificial biologics formats. Therefore, having a lineage directly diverging from the cell pool that was a transfectant from the cGMP-compliant facilities could suffice to both the needs of the rapid generation of consecutive batches of clinical materials (Gen1 production) and the critical classical clone screening and the monoclonal MCB generation (Gen2 activities). With the Gen1 production and the Gen2 development carried out in parallel, the Gen2 GMP batch materials from the monoclonal MCB will be available as soon as just one month after the second batch of the Gen1 GMP materials. The proximity of the critical quality attributes (CQA) between the Gen1 and the Gen2 is critically important, even though the wellestablished downstream process platform should be capable of removing common impurities, which should already be probed by the pilot transient expression study. This necessitates the CLD segment to screen the derivative clones extensively in search of the top clone candidates with both good proximity of CQA and excellent productivity. It is also advantageous if the upstream process segment could evaluate several chosen candidates on bioreactors, which could provide a more accurate performance evaluation for both the culturing and the purifying process. The rather painstaking clone selection would greatly help to minimize the need for process development, which could be very costly for time-sensitive pandemic projects. So far, the incorporation of advanced automation and high-throughput technology into our WuXia ${ }^{\mathrm{TM}}$ cell line development platform has made the cell screening effort much less labor-intensive than ever, which helps us to find a desirable clone from thousands of clone candidates in a highly tensed CMC context.

In the pharmaceutical industry, safety shall never be compromised regardless. After the decision of the MCB clone, there will be a set of biosafety tests for the RCBs or pre-MCBs before they are permitted to enter the cGMP facilities. The full set of biosafety testing typically takes at least 6-8 weeks to conclude, which is very difficult to maneuver in an aggressive CMC context. However, unwarranted clearance into the 
cGMP facilities of an RCB or pre-MCB, usually generated under non-cGMP condition, is going to stake the ongoing or forthcoming business activity of cGMP facilities, which could be egregiously costly for both the pandemic project and the core business interests. To solve this problem, we adopt the pre-warning NGS-based screening to provide a precautionary biosafety check for the RCBs, which should suffice with very scarce risks of contamination from the current industrial practice and experience. Comparing with the typical biological assays, the turnaround time for the NGS-based detection is just as short as one week, saving 5-7 weeks to continue the subsequent CMC activities.

To conclude, we have devised and successfully implemented a biologics development strategy spanning as short as 2.5 months from the top molecule decision to IND for several COVID-19 biologics. While the industry has been progressing from 18 months to 12 months or even less to IND, the outbreak of global pandemics, such as COVID-19, can still impose very threatening and tense situations to global public health. This needs our industry to come up with meaningful solutions to combat such an outbreak and protect public health. The successful execution of this unprecedentedly expedited CMC and manufacturing strategy relies very closely on the experiences accumulated from hundreds of antibody molecules, the well-furnished platforms of development protocols, as well as the efficient communications between different functionalities within the CMC team. Even with the carefully intended preceding material generation steps, the whole team should still make careful and economical use of the raw materials, to blaze the trail for the incoming strategic planning and manufacturing. With hundreds of valuable successful precedents of CMC for biologics, the team has already developed an accurate sense of material planning and accumulated enough experience to avoid premature attempts on unnecessary and time-costly trial and error, which are very valuable for the industry against the global pandemic emergency.

\section{Acknowledgment}

We are grateful to Vir Biotechnology and all other clients to commission the development and manufacturing of their valuable lifesaving molecules to us, and for us to join the fight against the global threat of COVID-19. We faithfully hope for fast and rapid and positive clinical trial outcomes, and timely BLA approval of these therapeutic biologics. We also sincerely thank the development, manufacturing, and quality teams of WuXi Biologics for the seamless collaborations and the board of leadership for their great support and valuable suggestions.

\section{References}

Baum, A., Fulton, B. O., Wloga, E., Copin, R., Pascal, K. E., Russo, V., Giordano, S., Lanza, K., Negron, N., Ni, M., Wei, Y., Atwal, G. S., Murphy, A. J., Stahl, N., Yancopoulos, G. D., \& Kyratsous, C. A. (2020). Antibody cocktail to SARS-CoV-2 spike protein prevents rapid mutational escape seen with individual antibodies. Science (New York, N.Y.), 369(6506), 1014-1018. https://doi.org/10.1126/science.abd0831

Bolisetty, P., Tremml, G., Xu, S., \& Khetan, A. (2020). Enabling speed to clinic for monoclonal antibody programs using a pool of clones for IND-enabling toxicity studies. mAbs, 12(1), 1763727. https://doi.org/10.1080/19420862.2020.1763727 Brouwer, P., Caniels, T. G., van der Straten, K., Snitselaar, J. L., Aldon, Y., Bangaru, S., Torres, J. L., Okba, N., Claireaux, M., Kerster, G., Bentlage, A., van Haaren, M. M., Guerra, D., Burger, J. A., Schermer, E. E., Verheul, K. D., van der Velde, N., van der Kooi, A., van Schooten, J., van Breemen, M. J., ... van Gils, M. J. (2020). Potent neutralizing antibodies from COVID-19 patients define multiple targets of vulnerability. Science (New York, N.Y.), $369(6504), 643-650$. https://doi.org/10.1126/science.abc5902 russel, A., Brack, K., Muth, E., Zirwes, R., Cheval, J., Hebert, C., Charpin, J. M., Marinaci, A., Flan, B., Ruppach, H., Beurdeley, P., \& Eloit, M. (2019). Use of a new RNA next generation sequencing approach for the specific detection of virus infection in cells. Biologicals : journal of the International Association of Biological Standardization, 59, 29-36. https://doi.org/10.1016/j.biologicals.2019.03.008 Cao, Y., Su, B., Guo, X., Sun, W., Deng, Y., Bao, L., Zhu, Q., Zhang, X., Zheng, Y., Geng, C., Chai, X., He, R., Li, X., Lv, Q., Zhu, H., Deng, W., Xu, Y., Wang, Y., Qiao, L., ... Xie, X. S. (2020). Potent Neutralizing Antibodies against SARS-CoV-2 Identified by High-Throughput Single-Cell Sequencing of Convalescent Patients' B Cells. Cell, 182(1), 73e16. https://doi.org/10.1016/j.cell.2020.05.025 Fan, L., Rizzi, G., Bierilo, K., Tian, J., Yee, J. C., Russell, 
R., \& Das, T. K. (2017). Comparative study of therapeutic antibody candidates derived from mini-pool and clonal cell lines. Biotechnology progress, 33(6), 1456-1462. https://doi.org/10.1002/btpr.2477 Gao, Q., Bao, L., Mao, H., Wang, L., Xu, K., Yang, M., Li, Y., Zhu, L., Wang, N., Lv, Z., Gao, H., Ge, X., Kan, B., Hu, Y., Liu, J., Cai, F., Jiang, D., Yin, Y., Qin, C., Li, J., .. Qin, C. (2020). Development of an inactivated vaccine candidate for SARS-CoV-2. Science (New York, N.Y.), 369(6499), 7781. https://doi.org/10.1126/science.abc1932 Hansen, J., Baum, A., Pascal, K. E., Russo, V., Giordano, S., Wloga, E., Fulton, B. O., Yan, Y., Koon, K., Patel, K., Chung, K. M., Hermann, A., Ullman, E., Cruz, J., Rafique, A., Huang, T., Fairhurst, J., Libertiny, C., Malbec, M., Lee, W. Y., ... Kyratsous, C. A. (2020). Studies in humanized mice and convalescent humans yield a SARS-CoV-2 antibody cocktail. Science (New York, N.Y.), $369(6506)$, 1010-1014. https://doi.org/10.1126/science.abd0827 He, L., Winterrowd, C., Kadura, I., \& Frye, C. (2012). Transgene copy number distribution profiles in recombinant $\mathrm{CHO}$ cell lines revealed by single cell analyses. Biotechnology and bioengineering, 109(7), 1713-1722. https://doi.org/10.1002/bit.24428 Hu, Z., Hsu, W., Pynn, A., Ng, D., Quicho, D., Adem, Y., Kwong, Z., Mauger, B., Joly, J., Snedecor, B., Laird, M. W., Andersen, D. C., \& Shen, A. (2017). A strategy to accelerate protein production from a pool of clones in Chinese hamster ovary cells for toxicology studies. Biotechnology progress, 33(6), 1449-1455. https://doi.org/10.1002/btpr.2467 Ju, B., Zhang, Q., Ge, J., Wang, R., Sun, J., Ge, X., Yu, J., Shan, S., Zhou, B., Song, S., Tang, X., Yu, J., Lan, J., Yuan, J., Wang, H., Zhao, J., Zhang, S., Wang, Y., Shi, X., Liu, L., .. Zhang, L. (2020). Human neutralizing antibodies elicited by SARS-CoV-2 infection. Nature, 584(7819), 115-119. https://doi.org/10.1038/s41586-020-2380-z Kelley B. (2020). Developing therapeutic monoclonal antibodies at pandemic pace. Nature biotechnology, 38(5), 540-545. https://doi.org/10.1038/s41587-020-0512-5 Khan, A. S., Benetti, L., Blumel, J., Deforce, D., Egan, W. M., Knezevic, I., Krause, P. R., Mallet, L., Mayer, D., Minor, P. D., Neels, P., \& Wang, G. (2018). Report of the international conference on next generation sequencing for adventitious virus detection in biologicals. Biologicals : journal of the International Association of Biological Standardization, 55, 1-16. https://doi.org/10.1016/j.biologicals.2018.08.002 Khan, A. S., Vacante, D. A., Cassart, J. P., Ng, S. H., Lambert, C., Charlebois, R. L., \& King, K. E. (2016). Advanced Virus Detection Technologies Interest Group (AVDTIG): Efforts on High Throughput Sequencing (HTS) for Virus Detection. PDA journal of pharmaceutical science and technology, 70 (6), 591-595. https://doi.org/10.5731/pdajpst.2016.007161 Kunert, R., \& Reinhart, D. (2016). Advances in recombinant antibody manufacturing. Applied microbiology and biotechnology, 100(8), 3451-3461. https://doi.org/10.1007/s00253-016-7388-9 Lan, J., Ge, J., Yu, J., Shan, S., Zhou, H., Fan, S., Zhang, Q., Shi, X., Wang, Q., Zhang, L., \& Wang, X. (2020). Structure of the SARS-CoV-2 spike receptor-binding domain bound to the ACE2 receptor. Nature, 581(7807), 215-220. https://doi.org/10.1038/s41586-020-2180-5 Li, F., Vijayasankaran, N., Shen, A. Y., Kiss, R., \& Amanullah, A. (2010). Cell culture processes for monoclonal antibody production. mAbs, 2(5), 466-479. https://doi.org/10.4161/mabs.2.5.12720 Munro, T. P., Le, K., Le, H., Zhang, L., Stevens, J., Soice, N., Benchaar, S. A., Hong, R. W., \& Goudar, C. T. (2017). Accelerating patient access to novel biologics using stable pool-derived product for non-clinical studies and single clone-derived product for clinical studies. Biotechnology progress, 33(6), 1476-1482. https://doi.org/10.1002/btpr.2572 Nyon, M. P., Du, L., Tseng, C. K., Seid, C. A., Pollet, J., Naceanceno, K. S., Agrawal, A., Algaissi, A., Peng, B. H., Tai, W., Jiang, S., Bottazzi, M. E., Strych, U., \& Hotez, P. J. (2018). Engineering a stable CHO cell line for the expression of a MERScoronavirus vaccine antigen. Vaccine, 36(14), 1853-1862. https://doi.org/10.1016/j.vaccine.2018.02.065 Pinto, D., Park, Y. J., Beltramello, M., Walls, A. C., Tortorici, M. A., Bianchi, S., Jaconi, S., Culap, K., Zatta, F., De Marco, A., Peter, A., Guarino, B., Spreafico, R., Cameroni, E., Case, J. B., Chen, R. E., Havenar-Daughton, C., Snell, G., Telenti, A., Virgin, H. W., .. Corti, D. (2020). Crossneutralization of SARS-CoV-2 by a human monoclonal SARS-CoV antibody. Nature, 583(7815), 290-295. https://doi.org/10.1038/s41586-020-2349-y Plavsic, M., Shick, K., Bergmann, K. F., \& Mallet, L. (2016). Vesivirus 2117: Cell line infectivity range and effectiveness of amplification of a potential adventitious agent in cell culture used for biological production. Biologicals : journal of the International Association of Biological Standardization, 44(6), 540-545. https://doi.org/10.1016/j.biologicals.2016.08.001 Rajendra, Y., Balasubramanian, S., McCracken, N. A., Norris, D. L., Lian, Z., Schmitt, M. G., Frye, C. C., \& Barnard, G. C. (2017). Evaluation of piggyBac-mediated CHO pools to enable material generation to support GLP 
toxicology studies. Biotechnology progress, 33(6), 1436-1448. https://doi.org/10.1002/btpr.2495 Richards, B., Cao, S., Plavsic, M., Pomponio, R., Davies, C., Mattaliano, R., Madden, S., Klinger, K., \& Palermo, A. (2014). Detection of adventitious agents using next-generation sequencing. PDA journal of pharmaceutical science and technology, 68(6), 651-660. https://doi.org/10.5731/pdajpst.2014.01025 Rogers, T. F., Zhao, F., Huang, D., Beutler, N., Burns, A., He, W. T., Limbo, O., Smith, C., Song, G., Woehl, J., Yang, L., Abbott, R. K., Callaghan, S., Garcia, E., Hurtado, J., Parren, M., Peng, L., Ramirez, S., Ricketts, J., Ricciardi, M. J., ... Burton, D. R. (2020). Isolation of potent SARS-CoV-2 neutralizing antibodies and protection from disease in a small animal model. Science (New York, N.Y.), 369(6506), 956-963. https://doi.org/10.1126/science.abc7520 Scarcelli, J. J., Shang, T. Q., Iskra, T., Allen, M. J., \& Zhang, L. (2017). Strategic deployment of CHO expression platforms to deliver Pfizer's Monoclonal Antibody Portfolio. Biotechnology progress, 33(6), 1463-1467. https://doi.org/10.1002/btpr.2493 Sempowski, G. D., Saunders, K. O., Acharya, P., Wiehe, K. J., \& Haynes, B. F. (2020). Pandemic Preparedness: Developing Vaccines and Therapeutic Antibodies For COVID-19. Cell, 181(7), 1458-1463. https://doi.org/10.1016/j.cell.2020.05.041 Shi, R., Shan, C., Duan, X., Chen, Z., Liu, P., Song, J., Song, T., Bi, X., Han, C., Wu, L., Gao, G., Hu, X., Zhang, Y., Tong, Z., Huang, W., Liu, W. J., Wu, G., Zhang, B., Wang, L., Qi, J., .. Yan, J. (2020). A human neutralizing antibody targets the receptor-binding site of SARS-CoV-2. Nature, 584(7819), 120-124. https://doi.org/10.1038/s41586-020-2381-y Wang, C. J., Feng, S. F., \& Duncan, P. (2014). Defining a sample preparation workflow for advanced virus detection and understanding sensitivity by next-generation sequencing. PDA journal of pharmaceutical science and technology, 68(6), 579-588. https://doi.org/10.5731/pdajpst.2014.01010 Wang, Q., Zhang, Y., Wu, L., Niu, S., Song, C., Zhang, Z., Lu, G., Qiao, C., Hu, Y., Yuen, K. Y., Wang, Q., Zhou, H., Yan, J., \& Qi, J. (2020). Structural and Functional Basis of SARS-CoV-2 Entry by Using Human ACE2. Cell, 181(4), 894-904.e9. https://doi.org/10.1016/j.cell.2020.03.045 Wright, C., Alves, C., Kshirsagar, R., Pieracci, J., \& Estes, S. (2017). Leveraging a CHO cell line toolkit to accelerate biotherapeutics into the clinic. Biotechnology progress, 33(6), 1468-1475. https://doi.org/10.1002/btpr.2548 Zhang, L., Lin, D., Sun, X., Curth, U., Drosten, C., Sauerhering, L., Becker, S., Rox, K., \& Hilgenfeld, R. (2020). Crystal structure of SARSCoV-2 main protease provides a basis for design of improved $\alpha$-ketoamide inhibitors. Science (New York, N.Y.), 368(6489), 409-412. https://doi.org/10.1126/science.abb3405

Figure Legends

\section{Table 1. Demonstration of NGS for virus detection.}

Figure 1. Gen1 and Gen2 overall workflow with SSI technology. SSI pools are employed for toxicology and early GMP production runs. The final clone MCB is used for the rest of GMP production.

Figure 2. Toxicology production from several COVID-19 molecules. (A): The titer data of some representative toxicology production of COVID-19 mAb molecules. (B-F): Viability, VCD, glucose level, lactate level and osmolality of Molecule D.

Figure 3. Comparable product quality among different batches of pool production. One representative COVID-19 pool to support toxicology run and two consecutive GMP runs with similar titer (A) and product quality (B-D) as its 3L bioreactor evaluation.

Figure 4. Clone selection from SSI pools. The titer (A), critical glycan species (B) and charge variants (C) data of toxicology, GMP and top clone candidates are charted. Key product quality attributes of Gen1 and Gen2 are comparable (D).

Figure 5. Preliminary cell line stability study.Expression levels (blue and orange bars) and titer variation (grey line) between RCB and PDL30 cells.

Figure 6. Representative product quality comparison. (A) SEC (B) charge variants (C) glycan comparison of one molecule among transient expression, toxicology, Gen1 GMP production, top clone candidates in AMBR, the final clone in 50L pilot, 500L pilot and 2000L GMP production. (D) Titer (E) SEC (F) charge variants $(\mathrm{C})$ glycan comparison of one another molecule among toxicology, Gen1 GMP production, 
Gen2 clonal bioreactor runs at different scales (3L, 15L and 500L GMP).

Figure 7. Representative timeline comparison. (A) A classical accelerated 12-month CMC timeline illustration. (B) The envisaged 6-month CMC timeline illustration.

Table 1 Demonstration of NGS for virus detection.

\begin{tabular}{llllll}
\hline Entry & Transfected cell counts $\left(\mathbf{1 0}^{\mathbf{6}}\right)$ & Host Cell & & \\
Counts $\left(10^{6}\right)$ & HC Read Counts/Base & LC Read Counts/Base & & \\
1 & 1.000 & 0 & 6690 & 5900 \\
2 & 0.333 & 0.667 & 5393 & 4965 \\
3 & 0.100 & 0.900 & 3491 & 3927 \\
4 & 0.033 & 0.967 & 825.5 & 1288 \\
5 & 0.010 & 0.990 & 258.3 & 514.3 \\
6 & 0 & 1.000 & ND & ND \\
\hline
\end{tabular}

Figure 1

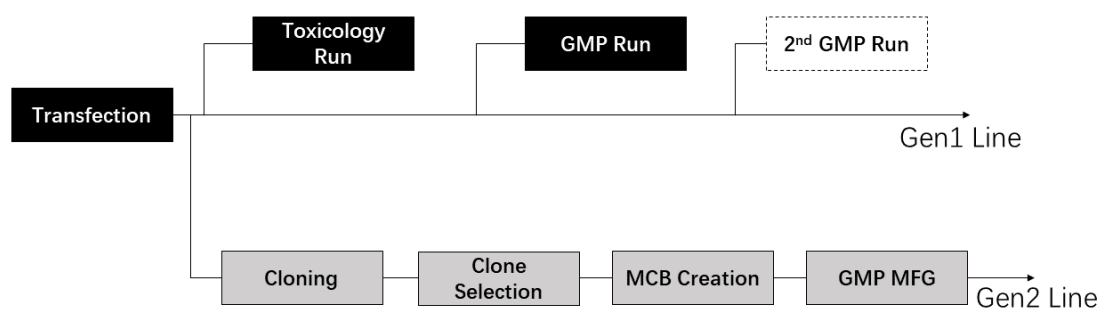

Figure 1. Gen1 and Gen2 overall workflow with SSI technology. SSI pools are employed for toxicology and early GMP production runs. The final clone MCB is used for the rest of GMP production.

Figure 2. 

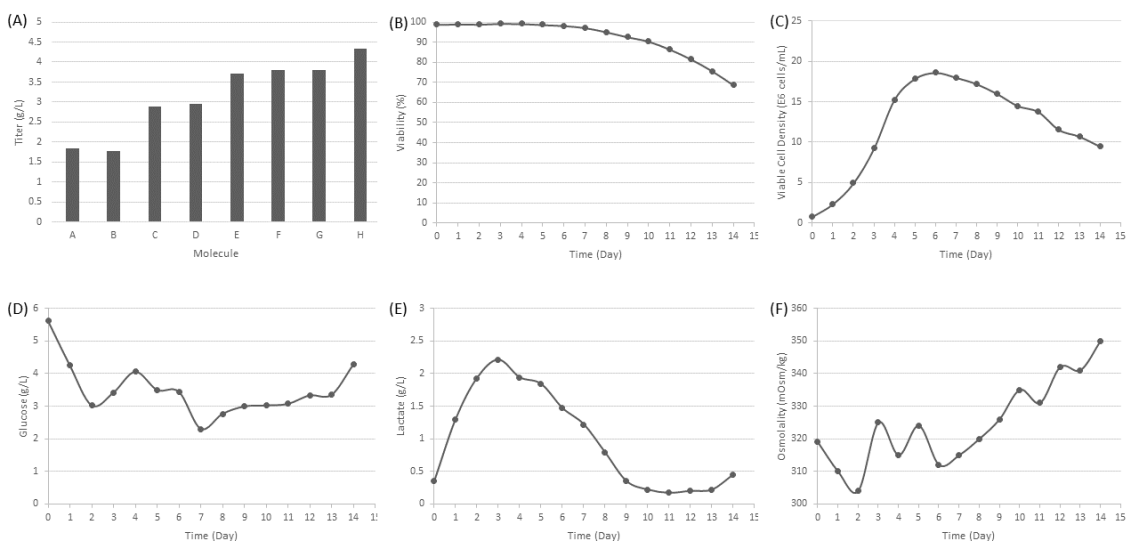

Figure 2. Toxicology production from several COVID-19 molecules. (A): The titer data of some representative toxicology production of COVID-19 mAb molecules. (B-F): Viability, VCD, glucose level, lactate level and osmolality of Molecule D.

\section{Figure 3}
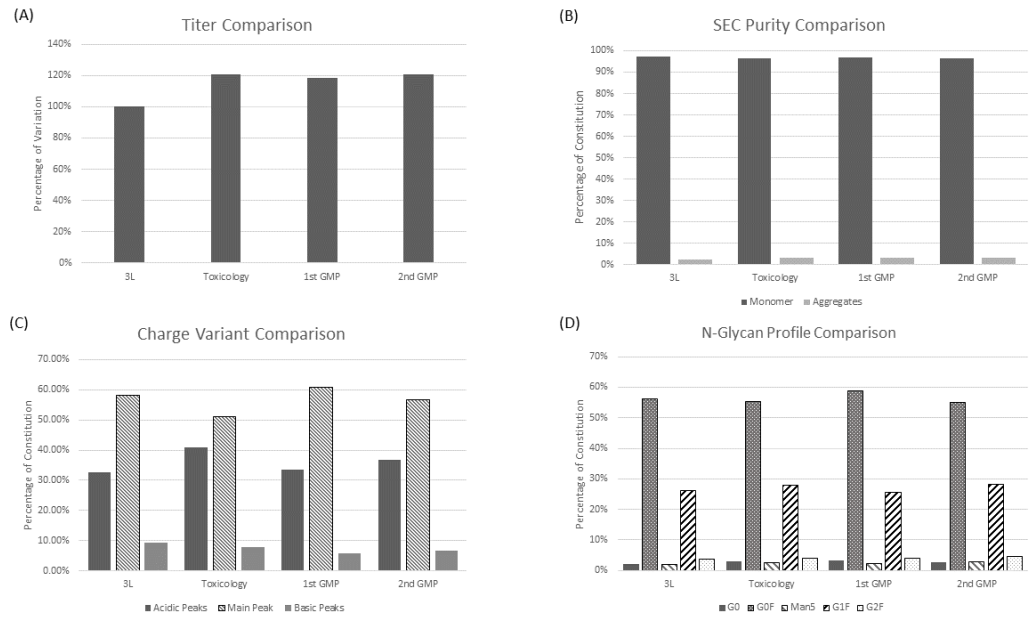

Figure 3. Comparable product quality among different batches of pool production. One representative COVID-19 pool to support toxicology run and two consecutive GMP runs with similar titer (A) and product quality (B-D) to its 3L bioreactor evaluation.

Figure 4 

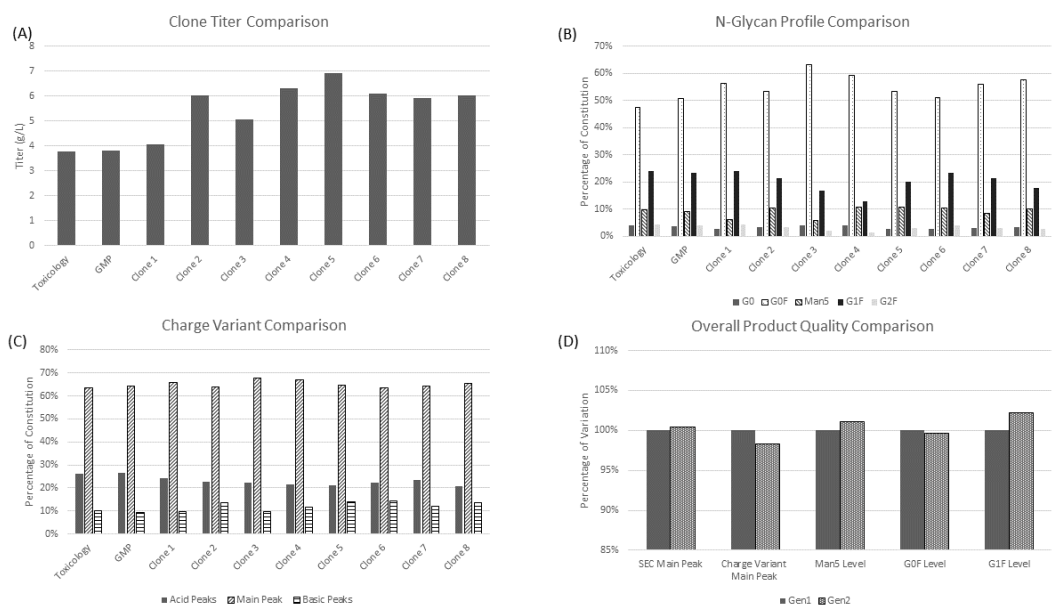

Figure 4. Clone selection from SSI pools. The titer (A), critical glycan species (B) and charge variants (C) data of toxicology, GMP and top clone candidates are charted. Key product quality attributes of Gen1 and Gen2 are comparable (D).

Figure 5

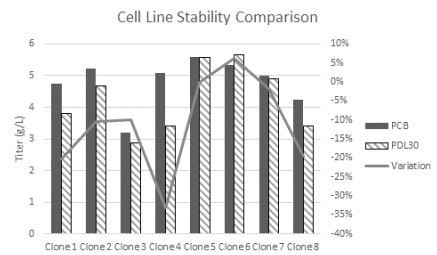

Figure 5. Preliminary cell line stability study.Expression levels (bars) and titer variation (line) between PCB and PDL30 cells.

Figure 6 

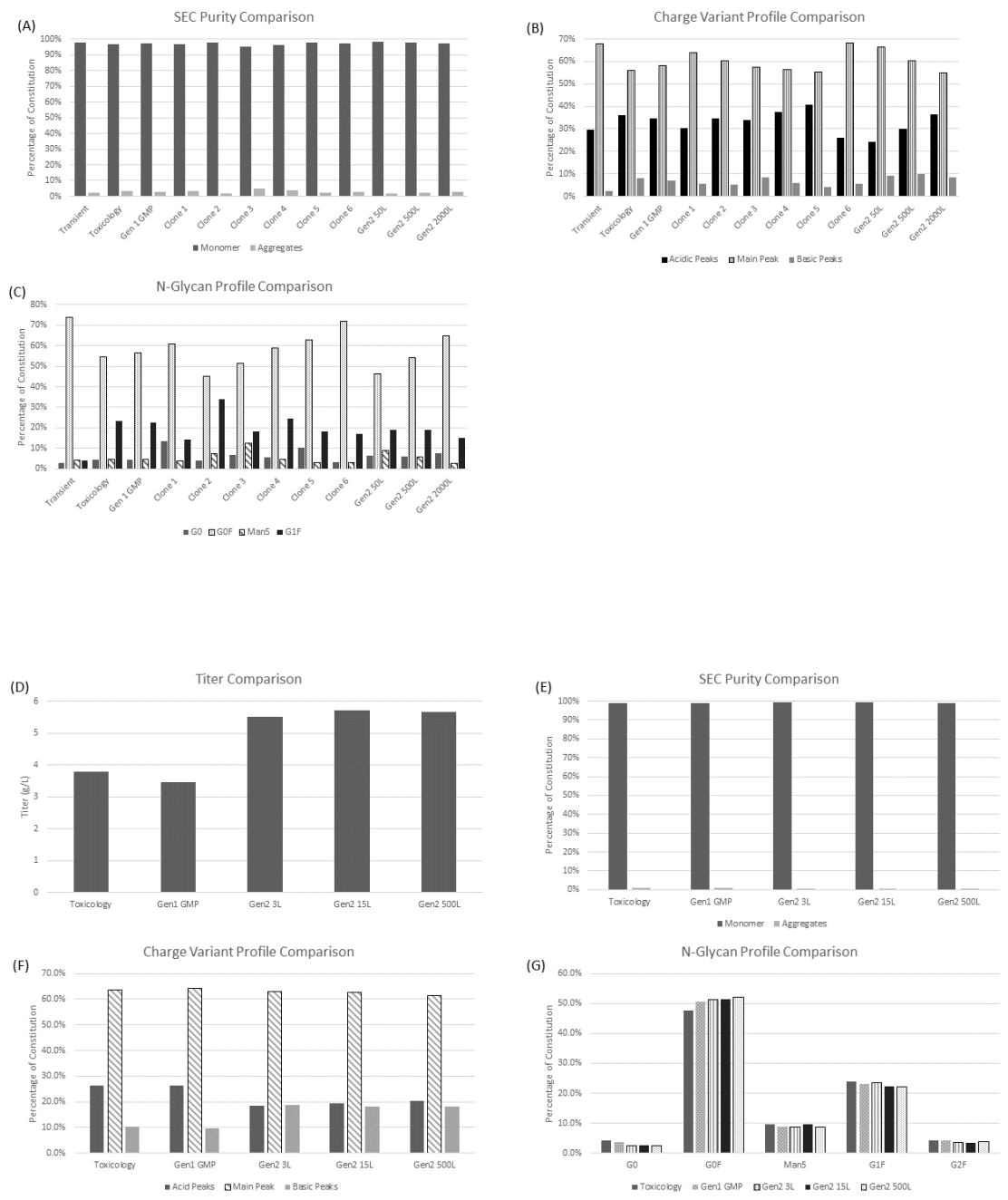

Figure 6. Representative product quality comparison. (A) SEC (B) charge variants (C) glycan comparison of one molecule among transient expression, toxicology, Gen1 GMP production, top clone candidates in AMBR, the final clone in 50L pilot, 500L pilot and 2000L GMP production. (D) Titer (E) SEC (F) charge variants $(\mathrm{G})$ glycan comparison of another molecule among toxicology, Gen1 GMP production, Gen2 clonal bioreactor runs at different scales (3L, 15L and 500L GMP).

\section{Figure 7}


(A)

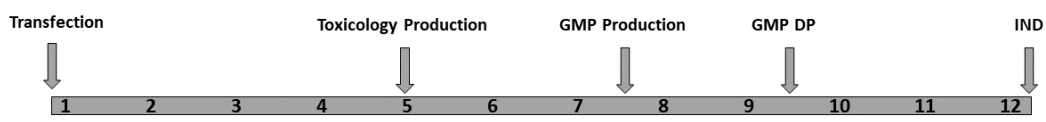

(B)

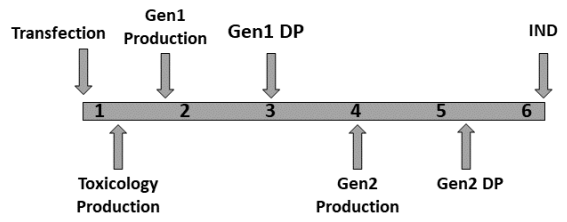

Figure 7. Representative timeline comparison. (A) A classical accelerated 12-month CMC timeline illustration. (B) The envisaged 6-month CMC timeline illustration.

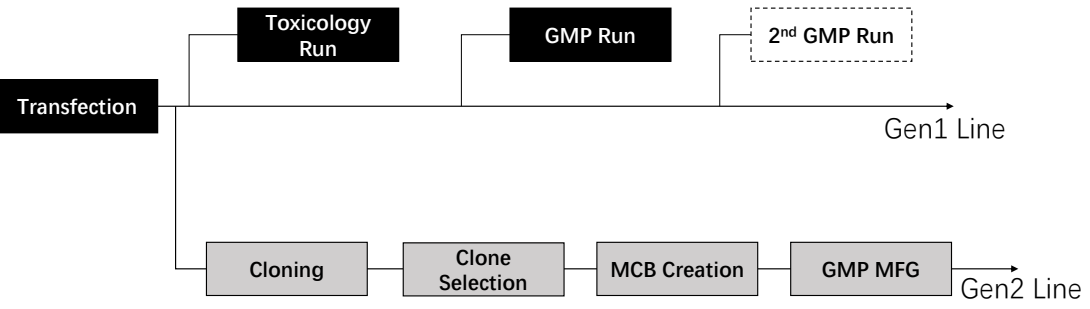



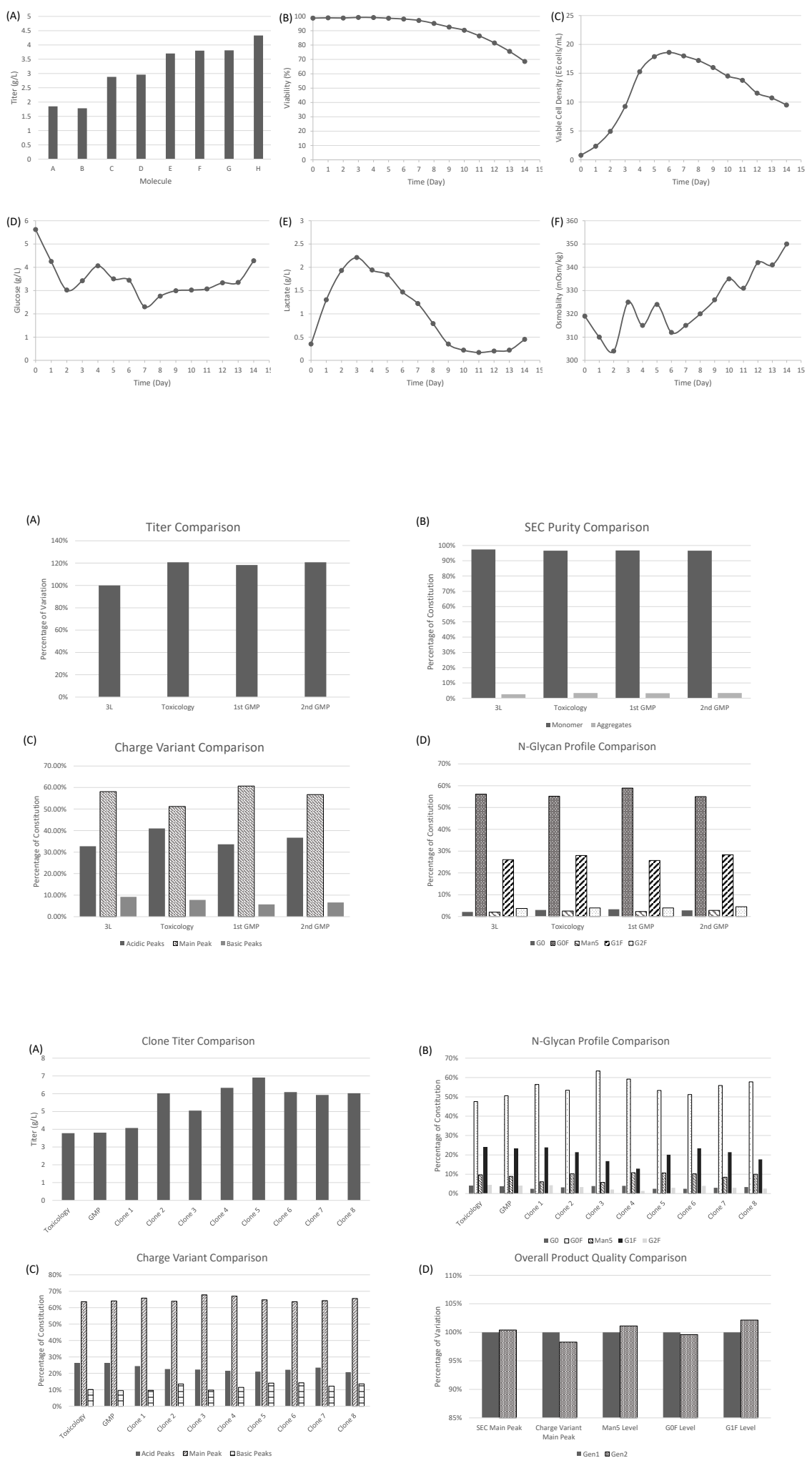

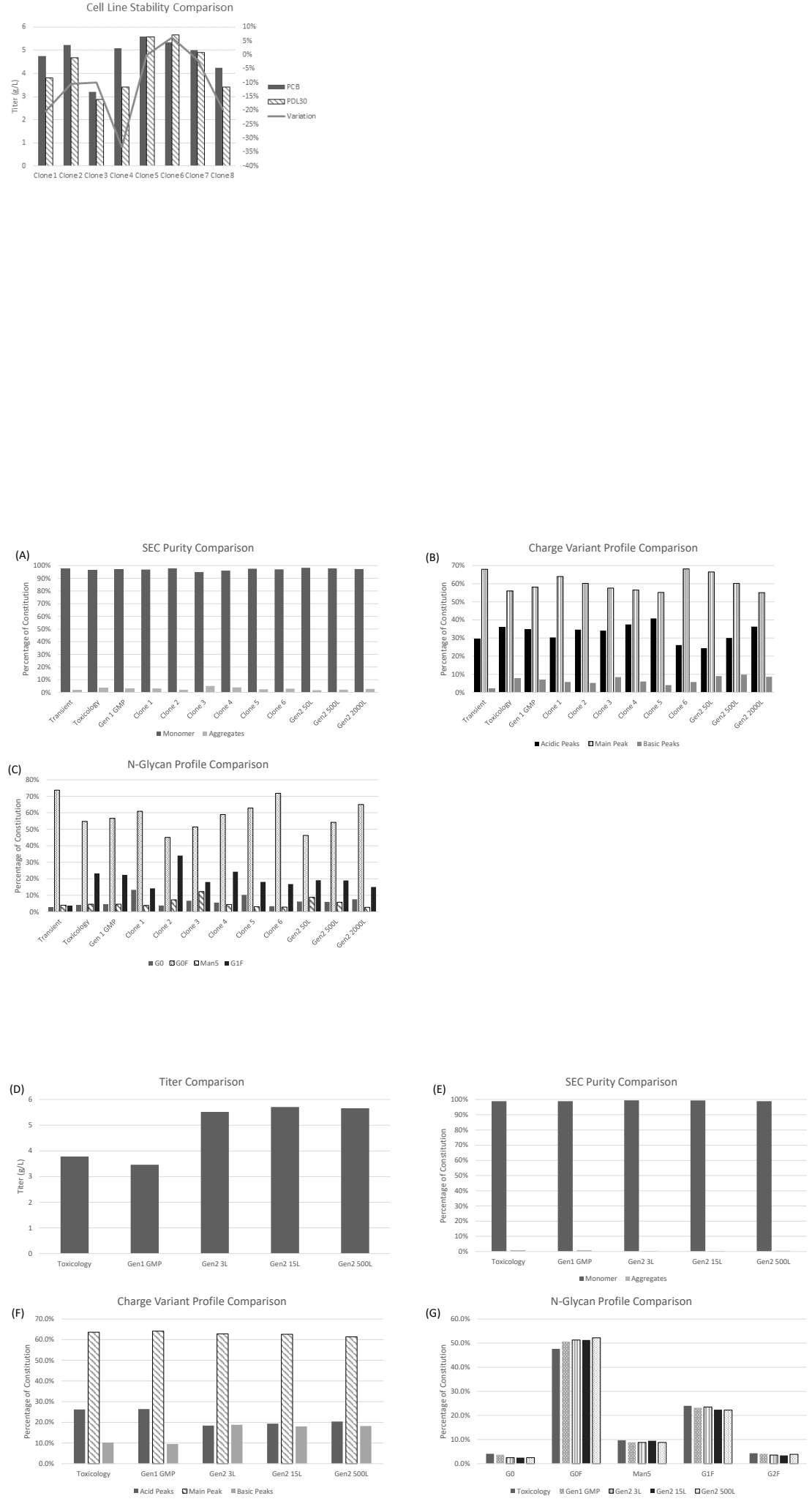
(A)

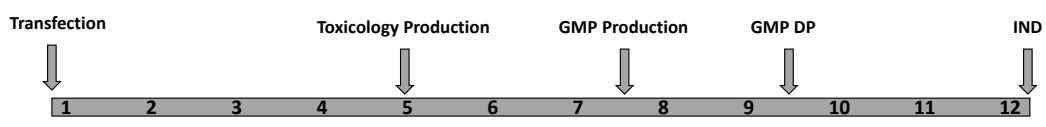

(B)

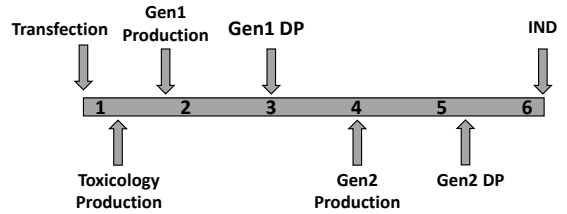

